\title{
Effect of semaphorin $3 A$ expression on clinicopathological features and prognosis in oropharyngeal carcinoma
}

Hai Thanh Pham

Kanazawa Daigaku https://orcid.org/0000-0003-3738-6229

Satoru Kondo ( $\nabla$ ksatoru@med.kanazawa-u.ac.jp )

Kazuhira Endo

Kanazawa Daigaku

Naohiro Wakisaka

Kanazawa Daigaku

Yoshitaka Aoki

Kanazawa Daigaku

Yosuke Nakanishi

Kanazawa Daigaku

Kina Kase

Kanazawa Daigaku

Harue Mizokami

Kanazawa Daigaku

Makoto Kano

Kanazawa Daigaku

Takayoshi Ueno

Kanazawa Daigaku

Miyako Hatano

Kanazawa Daigaku

Makiko Moriyama Kita

Kanazawa Daigaku

Hisashi Sugimoto

Kanazawa Daigaku

Tomokazu Yoshizaki

Kanazawa Daigaku

Research article

Keywords: SEMA3A, oropharyngeal carcinoma, neoangiogenesis, prognosis, MVD 
Posted Date: April 7th, 2020

DOl: https://doi.org/10.21203/rs.3.rs-21196/v1

License: (c) (i) This work is licensed under a Creative Commons Attribution 4.0 International License. Read Full License 


\section{Abstract}

Semaphorin 3A (SEMA3A) is a well-known axon guidance molecule in the nervous system. It is also known as a tumor suppressor in various cancers. In the present study, we examined the relationships between SEMA3A and clinicopathologic features and neoangiogenesis and its prognostic significance for oropharyngeal cancer (OPC) patients. Thirty-two OPC patients who underwent biopsy and 17 normal patients who underwent tonsillectomy were analyzed for SEMA3A expression by immunohistochemical analysis. We also analyzed 22 OPC specimens for CD34 expression as a marker of neoangiogenesis. SEMA3A was significantly downregulated in OPC compared with normal tonsil tissues $(p=0.005)$. SEMA3A expression was negatively correlated with CD34 expression, which suggested that a higher microvascular density corresponded to a lower expression of SEMA3A $(r=-0.466, p=0.033)$. Moreover, the higher SEMA3A expression cohort showed better survival than the lower SEMA3A expression cohort regardless of human papillomavirus (HPV) status $(p=0.035)$. These results suggest that SEMA3A expression is a prognostic marker for survival and is associated with antiangiogenesis in OPC.

\section{Background}

Head and neck cancer (HNC) is the 6th most prevalent cancer worldwide and is considered the sixth leading cause of cancer mortality [1]. Oropharyngeal cancer (OPC) has become the common subsite of $\mathrm{HNC}$, oral sex has been believed to be involved in the development of Human papillomavirus (HPV)induced OPC [2]. OPC incidence has increased over the last 20 years in several countries [3] [4] [5] [6], including the United States [7], Canada [8], and Japan [9]. Nearly all cases of cervical cancers can be attributable to HPV infection, in contrast, OPC has two distinct etiologies: tobacco and alcohol consumption, and HPV infection [10]. Making this distinction is clinically important because HPV(+) OPC has a more favorable prognosis compared with OPC that is HPV(-) [11] [12] [13] [14] [15].

Semaphorins are a large family of axon guidance. Semaphorins include both secreted and membranebound proteins that were initially implicated in the development of the nervous system and axon guidance. Recently, Semaphorin 3A (SEMA3A) has been shown to regulates cell adhesion, cell motility, angiogenesis, immune responses, and tumor progression [16] [17]. In the mammalian system, 20 semaphorins have been grouped into five classes (semaphorins $3-7$ ), in which class 3 semaphorins are secreted proteins and classes 4-6 semaphorins are transmembrane proteins. SEMA3A is identified as a potent tumor suppressor in some cancers (breast cancer and prostate cancer [18][19]), inhibits endothelial cell adhesion and migration [20], induces the collapse of the actin cytoskeleton and apoptosis, reduces angiogenesis in vitro[21]. Overexpression of SEMA3A is believed to inhibit angiogenesis and tumor growth in different mouse allograft and xenograft models [22] [23]. The expression levels of SEMA3A are also overexpressed in meningiomas tissues, and the expression level is negatively correlated with the microvessel density (MVD) of the tumor [24]. MVD has also been determined to be an effective prognostic factor and is related to survival in OPC [25]. 
However, until now, the effect of SEMA3A in OPC had not been studied intensively and remained unclear. Therefore, we focused on the expression of SEMA3A in OPC to determine the clinical significance of SEMA3A expression, especially its prognostic significance for the survival of OPC patients and its correlation with MVD, in this study.

\section{Materials And Methods}

\section{Patients and tissue samples}

From June 2004 to February 2015, the biopsy specimens of 32 OPC patients who underwent biopsy and 17 normal patients who underwent tonsillectomy were obtained from the Division of OtolaryngologyHead and Neck Surgery, Kanazawa University Hospital. Thirty-two cancer biopsy specimens were obtained from OPC patients and then verified by pathological sectioning. None of these patients had received radiotherapy or chemotherapy prior to biopsy. Seventeen noncancerous tonsil tissues were obtained from normal patients who had tonsillitis. All tissues were obtained with the consent of patients. Clinical information related to 33 cancer patients, including age, sex, and TNM stage, was also obtained. The TNM stage was defined by the guidelines of the UICC, 8th edition, 2018. Distant metastasis was determined by radiological examination. Overall survival, which was defined as the time from diagnosis to the time of patient death, date of censoring due to loss of follow-up or last follow-up, was used as a measure of prognosis and ranged from 2 to 121 months. This study was approved by the Ethics Committee of Kanazawa University.

\section{Immunohistochemistry}

Among the 32 OPC biopsy specimens, all 32 were immunohistochemically examined for SEMA3A, and 22 were examined for CD34. Tumor angiogenesis is an important step in tumor growth and metastasis. Antibodies against CD34, which is specific to the vein endothelium, were used for MVD evaluation [25]. All specimens were fixed in a 10\% formaldehyde solution and embedded in paraffin. Tissues were deparaffinized in xylene and rehydrated through an alcohol gradient. Endogenous peroxidase activity was blocked with $3 \%$ hydrogen peroxide for 10 min after 3 washes in phosphate-buffered saline at pH 7.2. The slides were boiled in $10 \mathrm{mM}$ sodium citrate retrieval buffer $(\mathrm{pH} \mathrm{6.0)}$ for $20 \mathrm{~min}$ for antigen retrieval. After rinsing with PBS, the tissue sections were incubated with a protein block (Dako, Glostrup, Denmark) for 20 min and incubated overnight at $4{ }^{\circ} \mathrm{C}$ with the following antibodies as the primary antibody: rabbit antiSEMA3A (1:200, Abcam, Cambridge, UK) and rabbit anti-CD34 (1:100, Abcam, Cambridge, UK). The sections were washed three times with PBS. Next, the sections were exposed to EnVision + secondary antibody (Dako) for $30 \mathrm{~min}$. The reaction products were developed by immersing the sections in 3',3diaminobenzidine tetrahydrochloride solution. Subsequently, the sections were counterstained with hematoxylin. The stained sections were independently evaluated by two investigators (H. P. and S. K.) who were blinded to the clinical data using an IX83 microscope (Olympus, Tokyo, Japan).

\section{Evaluation of SEMA3A Immunoreactivity}


Each slide was observed by scanning the whole section at medium $(\times 40)$ and high $(\times 200)$ magnification under a light microscope. The immunoreactive score was defined as the proportion score multiplied by the intensity score. The criteria were as follows: i) The intensity of SEMA3A staining was scored as 0 (negative), 1 (weak), 2 (medium), or 3 (strong); ii) the percentage of positive-staining cancer cells in tumor specimens or positive-staining epithelial cells in the specimens was scored as $0(0-5 \%), 1(6-25 \%), 2$ $(26-50 \%), 3(51-75 \%)$, or $4(76-100 \%)$. The total score ranged from 0 to 12 . The expression of SEMA3A was divided into the following groups: Negative immunoreactivity was defined as a total score of $0, a$ score of 1-4 was low expression, and a score of $>4$ was high expression.

\section{Evaluation of CD34 Immunoreactivity}

The vascular objects were counted to determine vascular density (vessel $\left./ \mathrm{mm}^{2}\right)$. Ten areas of densely concentrated microvessels (hotspots) were located using $100 \times$ magnification (objective lens $\times 10$ and ocular lens $\times 10$ ). In each case, these hotspot areas were used for counting microvessels at $400 \times$ magnification ( $40 \times$ objective lens and $10 \times$ ocular lens). A vascular unit was identified according to the criteria established by Weidner [26], who described a vascular unit as a cell or group of endothelial cells of a brownish color clearly separated from adjacent microvessels, tumor cells, and other connective tissue. The total number of vessels obtained in each case was the result of the total sum of vessels counted in each of the 10 microscopic fields evaluated at $400 \times$ magnification. MVD was defined as the average number of microvessels per field, which was calculated from the total number of microvessels in 10 fields [27].

\section{Statistical analyses}

SPSS statistics package version 19 (IBM, New York, NY) was used for data analysis. The clinical characteristics of the patients were analyzed using Fisher's exact test and the chi-square test. The Spearman test was used in the correlation analysis. Overall survival was obtained using the Kaplan-Meier method. $P$ values less than 0.05 were considered statistically significant.

\section{Results}

\section{Immunohistochemical analysis of SEMA3A expression in OPC tissues}

Our study included 32 patients with OPC and 17 patients with normal tonsils for immunohistochemical analysis. SEMA3A was detected primarily in the cytoplasm of tumor cells and normal cells (Fig. 1A, B). Only 3 of 17 (17.64\%) normal tonsil tissues showed low SEMA3A expression, whereas 19 of 32 (59.37\%) specimens of OPC tissue displayed low SEMA3A expression, indicating that SEMA3A expression was significantly upregulated in the OPC tissues compared with normal tonsil tissues $(p=0.005)$ (Table 1$)$.

\section{Association between the expression of SEMA3A and various clinicopathological features}


The chi-square test revealed that there was no significant difference in SEMA3A between the groups classified by age, sex, subsite, TNM stage, tumor stage, lymph node metastasis, HPV status, smoking history, and alcohol consumption (Table 2).

\section{Association between the expression of SEMA3A and survival}

We next assessed the relationship of SEMA3A expression with prognosis in OPC patients. Overall survival (OS) analysis was performed in the 32 patients, and the five-year OS rate was $66.5 \%$ (Fig. 2A). The fiveyear OS rate was $44.3 \%$ for patients with low SEMA3A expression and $83.3 \%$ for patients with high SEMA3A expression, which was a significant difference (Fig. 2B). Finally, we proved that the high SEMA3A expression group had a significantly longer survival than the low expression group. Next, by separating stages I-II and stages III-IV disease, we found that early-stage (stage I-II) disease with low levels of SEMA3A expression had a worse prognosis than early-stage disease with high levels of SEMA3A expression (Fig. 2C). In the samples of late-stage (stage III and IV) disease, the samples with low levels of SEMA3A expression also had a worse prognosis than the samples with high levels of SEMA3A expression (Fig. 2D). Regarding HPV infection, we also found that patients with low levels of SEMA3A expression had a worse prognosis than patients with high levels of SEMA3A regardless of HPV status. (Fig. 2E. F).

\section{Association between SEMA3A expression and MVD}

We next examined whether there was any correlation between the expression of SEMA3A and CD34 in OPC tissues using IHC analysis. IHC with CD34 antibody was performed on 22 OPC specimens (Fig. 3A). We revealed that the expression score of SEMA3A and the number of microvessels (CD34) in OPC samples were negatively correlated (Fig. 3B). These results support the hypothesis that SEMA3A acts as a tumor suppressor or angiogenesis inhibitor.

\section{Discussion}

The present study focused on the role of SEMA3A in the prognosis and pathological angiogenesis of OPC. SEMA3A has been identified as a candidate tumor suppressor and it is often found to be downregulated in different types of cancer, such as prostate cancer, breast cancer, and glioma. Increased SEMA3A expression was associated with better prognosis in patients with cancer, including epithelial ovarian carcinoma, gastric cancer, tongue cancer, and HNC [28] [29] [30] [31]. A reduction in SEMA3A expression was observed in our OPC samples compared with normal tonsil tissues.

Moreover, SEMA3A can inhibit the proliferation of malignant mesothelial cells, decrease the adhesion or migration of prostate or breast cancer cells, and promote apoptosis in leukemic T cells [32].

Overexpression of SEMA3A inhibits gastric cancer cell proliferation and migration in vitro [29]. The data presented here suggest that SEMA3A has a tumor suppressor function in OPC because our Kaplan-Meier 
survival analysis showed that low SEMA3A expression significantly correlated with shorter survival time in OPC patients. This result is consistent with earlier reports indicating that SEMA3A acts as an antitumorigenesis agent. To clarify the effect of SEMA3A, we analyzed survival by separating stages I-II and stages III-IV disease. This analysis indicated that patients with low levels of SEMA3A expression had a worse prognosis than those with high levels of SEMA3A expression in both early-stage and advancedstage disease. HPV infection has been increasingly recognized as an important etiological factor for a subset of HNCs, including OPC [33]. HPV(-) OPC carries a poorer prognosis compared with HPV(+) tumors [12]. The improved survival of patients with HPV (+) tumors can be attributed in part to their remarkable treatment sensitivity, as HPV (-) tumors have worse response to chemotherapy and radiation than HPV (+) tumors [13]. In our study, we found that low SEMA3A expression significantly correlated with decreased survival in OPC patients with or without HPV.

We next immunohistochemically analyzed SEMA3A expression and CD34 expression in the same tumor to evaluate the correlation of SEMA3A with the MVD. We found a significant association between high expression of SEMA3A and low MVD in the tumors. Tumor angiogenesis induces increased tumor cell circulation and it is an essential component of the metastatic pathway. An important evidence about the relationship between angiogenesis and metastasis is that tumor MVD leads to increased metastatic potential and poor survival in nearly all types of cancer [34]. Angiogenesis is a cancer hallmark, important for metastatic tumors, and is essential for the growth of lung micrometastases [34]. CD34 antibody is a credible marker for MVD evaluation. Recent studies have found associations between MVD and poor disease course in breast, lung, stomach, and bladder cancer [35] [36] [37].

Our results suggest that SEMA3A may be an antiangiogenic factor in OPC. The molecular mechanisms underlying the anti-tumor effects of SEMA3A are being extensively studied, the antiangiogenic factor SEMA3A may be capable of inhibiting the proangiogenic activity of vascular endothelial growth factor (VEGF) [38] [20]. Surprisingly, it is suggested that SEMA3A regulates a signaling pathway of its own because SEMA3A promotes vascular permeability, suppresses endothelial cell proliferation, and induces apoptosis when VEGF is not present [21] [39][40]. The proinvasive and prometastatic resistance detected upon angiogenesis reduction by the small-molecule tyrosine inhibitor sunitinib in pancreatic neuroendocrine tumors (PNETs) can be overwhelmed by SEMA3A expression [41]. Metastatic PNETs and cervical carcinomas are transformed into benign lesions by reexpressing SEMA3A, since SEMA3A not only enhanced cancer tissue oxygenation and increased the normalization window to inhibit sunitinibinduced activation of epithelial-mesenchymal transition (EMT) and hypoxia-dependent signaling pathways, such as the hypoxia-inducible factor 1-a related pathway, but also prevented tumor hypoxia and restrained cancer dissemination [41]. This logic may lead to the consideration of SEMA3A as a potential therapeutic for the treatment of cancer, specifically in combination with bevacizumab. Bevacizumab blocks the binding of VEGF to its cell surface receptors. This leads to a reduction in the development of vascular structures, as a result, the blood supply to tumor tissues is limited. These effects also reduce tissue interstitial pressure, promote vascular permeability, and support apoptosis of tumor endothelial cells. Increased tumor permeability may increase delivery of chemotherapeutic agents toward the tumor center [42]. Cell proliferation and colony formation were inhibited by SEMA3A and recombinant 
SEMA3A. Furthermore, intratumoral SEMA3A was believed to restrain tumor growth in a xenograft model [31]. In vitro, SEMA3A suppressed extracellular matrix-mediated adhesion and migration of endothelial cells [20].

In the present study, we found that the loss of SEMA3A expression in OPC and low expression of SEMA3A correlated with poor outcome in patients and high MVD in OPC samples. Taken together, our results demonstrate that SEMA3A serves as a tumor suppressor of OPC tumorigenesis and may become a new target for the treatment of OPC.

\section{Abbreviations}

SEMA3A

Semaphorin $3 A$

OPC

Oropharyngeal cancer

HNC

Head and neck cancer

HPV

Human papillomavirus

MVD

Microvessel density

UICC

Union for International Cancer Control

$\mathrm{IHC}$

Immunohistochemistry

VEGF

Vascular endothelial growth factor

PNETS

pancreatic neuroendocrine tumors

EMT

Epithelial-mesenchymal transition

\section{Declarations}

\section{Availability of data and materials}

The datasets used and/or analysed during the current study are available from the corresponding author on reasonable request

\section{Acknowledgments}


We thank our colleagues from the Division of Otolaryngology-Head and Neck Surgery, Kanazawa University Hospital, who provided insight and expertise that greatly assisted the research and and everyone who collected data and participated in the study.

This manuscript was edited for proper English language, grammar, punctuation, spelling and overall style by SpringerNature Author Services.

\section{Funding}

This study was supported by research grants from the Ministry of Education, Science, Sports, Culture and Technology of Japan (A24689064 for S. K. and B23390396 for T. Y.), and Yakult Grants for Research on Lactic Acid Bacteria for T. Y.

\section{Author information}

Division of Otolaryngology-Head and Neck Surgery, Graduate School of Medical Science, Kanazawa University, Kanazawa, Ishikawa, Japan

\section{Authors' contributions}

Conceptualization, S.K., and T.Y.; methodology, P.T.H., K.E., N.W.,Y.N, T.U.,H.M, M.K, investigation,Y.A, K.K., T.U., Y.N., M.H., H.S., M.M.-K.; supervision, S.K. and T.Y. All authors have read and agreed to the published version of the manuscript

\section{Ethic declarations}

This study was approved by the Ethics Committee of Kanazawa University

\section{Consent for publication}

Consent for publication was signed by all study participants.

\section{Competing interests}

The authors declare that they have no competing interests.

\section{References}

[1] L. A. Torre, F. Bray, R. L. Siegel, et al. Global cancer statistics, 2012. CA. Cancer J. Clin. 2015;65(2):87-108. doi: 10.3322/caac.21262.

[2] A. K. Chaturvedi, E. Engels, R.Pfeiffer, et al. Human papillomavirus and rising oropharyngeal cancer incidence in the United States. J. Clin. Oncol. 2011;29(32):4294-4301. doi: 10.1200/JC0.2011.36.4596. 
[3] A. Shanthi Marur, Gypsyamber D'Souza, William H. Westra, et al. HPV-associated Head and Neck Cancer: A Virus-related Cancer Epidemic - A Review of Epidemiology, Biology, Virus Detection and Issues in Management. Lancet Oncol. 2017;176(1):139-148. doi: 10.1016/j.physbeh.2017.03.040.

[4] T. Ramqvist and T. Dalianis. Oropharyngeal cancer epidemic and human papillomavirus. Emerg. Infect. Dis. 2010;16(11):1671-1677. doi: 10.3201/eid1611.100452.

[5] A. K. Chaturvedi. Epidemiology and Clinical Aspects of HPV in Head and Neck Cancers. Head Neck Pathol. 2012;6(SUPPL.1):16-24. doi: 10.1007/s12105-012-0377-0.

[6] M. L. Gillison, L. Alemany, P. Snijders, et al. Human papillomavirus and diseases of the upper airway: Head and neck cancer and respiratory papillomatosis. Vaccine 30. 2012(SUPPL.5):F34-F54. doi: 10.1016/j.vaccine.2012.05.070.

[7] A. Chaturvedi, E. Engels, W. Anderson, et al. Incidence trends for human papillomavirus-related and unrelated oral squamous cell carcinomas in the United States. J Clin Oncol. 2008;26(4):612-619. doi: $10.1200 / J C 0.2007 .14 .1713$

[8] A. Auluck, G. Hislop, C. Bajdik, et al. Trends in oropharyngeal

oral cavity cancer incidence of Human Papillomavirus (HPV)-related and HPV-unrelated sites in a multicultural population: The British Columbia experience. Cancer. 2010;116(11):2635-2644, , doi: 10.1002/cncr.25087.

[9] A. Chaturvedi, W. Anderson, J. Lortet Tieulent, et al. Worldwide trends in incidence rates for oral cavity and oropharyngeal cancers. J Clin Oncol. 2013;31(36):4550-4559. doi:10.1200/JC0.2013.50.3870.

[10] J. H. Maxwell, B. Kumar, F. Feng, et al. Tobacco use in HPV-positive advanced oropharynx cancer patients related to increased risk of distant metastases and tumor recurrence. Clin Cancer Res. 2010;16(4):1226, , doi: 10.1158/1078-0432.CCR-09-2350.

[11] F. Dayyani, C. J. Etzel, M. Liu, et al. Meta-analysis of the impact of human papillomavirus (HPV) on cancer risk and overall survival in head and neck squamous cell carcinomas (HNSCC). Head Neck Oncol. 2010;2(1):1-11, doi: 10.1186/1758-3284-2-15.

[12] K. K. Ang, J. Harris, R. Wheeler, et al. Human Papillomavirus and Survival of Patients with Oropharyngeal Cancer. N Engl J Med. 2010;363(1):24-35. doi: 10.1056/NEJMoa0912217.

[13] C. Fakhry, W. Westra, S. Li, et al. Improved survival of patients with human papillomavirus-positive head and neck squamous cell carcinoma in a prospective clinical trial. J. Natl. Cancer Inst. 2008;100( 4):261-269 . doi: 10.1093/jnci/djn011.

[14] H. M. Dahlstrand, D. Lindquist, L. Bjornestal, et al. P16INK4a correlates to human papillomavirus presence, response to radiotherapy and clinical outcome in tonsillar carcinoma. Anticancer Res. 
2005;25(6):4375-4383.

[15] F. P. Worden, B Kumar, J. Lee, et al. Chemoselection As a Strategy for Organ Preservation in Advanced Oropharynx Cancer: Response and Survival Positively Associated With HPV16 Copy Number. J Clin Onco. 2009;26(19): 3138-3146. doi: 10.1200/JC0.2007.12.7597.

[16] L. Tamagnone and P. M. Comoglio. To move or not to move? Semaphorin signalling in cell migration. EMBO Rep. 2004;5(4):356-361. doi: 10.1038/sj.embor.7400114.

[17] R. P. Kruger, J. Aurandt, and K. L. Guan. Semaphorins command cells to move. Nat. Rev. Mol. Cell Biol. 2005;6(10):789-800. doi: 10.1038/nrm1740.

[18] K. Li, M. K. Chen, L.Y. Li, et al. The predictive value of semaphorins 3 expression in biopsies for biochemical recurrence of patients with low- and intermediate-risk prostate cancer. Neoplasma. 2013;60(5):607-616, 2013. doi: 10.4149/neo.

[19] C. A. Staton, L. Shaw, M. Valluru, et al. Expression of class 3 semaphorins and their receptors in human breast neoplasia. Histopathology. 2011;59(2):274-282. doi: 10.1111/j.1365-2559.2011.03922.x.

[20] H. Q. Miao, S. Soker, L. Feiner, et al. Neuropilin-1 mediates collapsin-1/semaphorin III inhibition of endothelial cell motility: Functional competition of collapsin-1 and vascular endothelial growth factor165. J. Cell Biol. 1999;146(1): 233-241. doi: 10.1083/jcb.146.1.233.

[21] N. Guttmann-Raviv, N. Shraga-Heled, A. Varshavsky, et al. Semaphorin-3A and semaphorin-3F work together to repel endothelial cells and to inhibit their survival by induction of apoptosis. J. Biol. Chem. 2007;282(36):26294-26305. doi: 10.1074/jbc.M609711200.

[22] G. Chakraborty, S. Kumar, R. Mishra, et al. Semaphorin 3A suppresses tumor growth and metastasis in mice melanoma model. PLoS One. 2012;7(3):e33633. doi: 10.1371/journal.pone.0033633.

[23] B. Kigel, A. Varshavsky, O. Kessler, et al. Successful inhibition of tumor development by specific class-3 semaphorins is associated with expression of appropriate semaphorin receptors by tumor cells. PLoS One. 2008;3(9):e3287. doi: 10.1371/journal.pone.0003287.

[24] V. Barresi, E. Vitarelli, and S. Cerasoli. Semaphorin3A immunohistochemical expression in human meningiomas: Correlation with the microvessel density. Virchows Arch. 2009;454(5):563-571. doi: 10.1007/s00428-009-0757-3.

[25] E. Tastekin, V. Caloglu, N. Durankus, et al. Survivin expression, HPV positivity and microvessel density in oropharyngeal carcinomas and relationship with survival time. Arch. Med. Sci. 2017;13(6):1467-1473. doi: 10.5114/aoms.2015.56616.

[26] F. J. Weidner N, Semple JP, Welch WR, et al. Tumor angiogenesis and metastasis correlation in invasive breast carcinoma...N. Engl. J. Med. 1991;329(14):977-986, 1991, doi: 
[27] I. Kuzu, R. Bicknell, A. L. Harris, et al. Heterogeneity of vascular endothelial cells with relevance to diagnosis of vascular tumours. J. Clin. Pathol. 1992;45(2):143-148, 1992. doi: 10.1136/jcp.45.2.143.

[28] H. Jiang, L. Qi, F. Wang, Z. Sun, et al. Decreased semaphorin 3A expression is associated with a poor prognosis in patients with epithelial ovarian carcinoma. Int. J. Mol. Med. 2015;35(5):1374-1380. doi: 10.3892/ijmm.2015.2142.

[29] C. Tang, X. Gao, H. Liu, T. Jiang, et al. Decreased expression of SEMA3A is associated with poor prognosis in gastric carcinoma. Int. J. Clin. Exp. Pathol. 2014;7(8):4782-4794.

[30] X. Song, W. Zhang, Y. Zhang, et al. Expression of semaphorin 3A and neuropilin 1 with clinicopathological features and survival in human tongue cancer. Med. Oral Patol. Oral Cir. Bucal. 2012;17(6):e962-8. doi: 10.4317/medoral.18168.

[31] Z. Wang, J. Chen, W. Zhang, et al. Axon guidance molecule Semaphorin3A is a novel tumor suppressor in head and neck squamous cell carcinoma. Oncotarget. 2016;7(5):6048-6062, 2016. doi: 10.18632/oncotarget.6831.

[32] M. Yacoub, A. Coulon, O. Celhay, et al. Differential expression of the semaphorin 3A pathway in prostatic cancer. Histopathology. 2009;55(4):392-398. doi: 10.1111/j.1365-2559.2009.03406.x.

[33] S. Elrefaey, M. A. Massaro, S. Chiocca, et al. HPV in oropharyngeal cancer: the basics to know in clinical practice. Acta Otorhinolaryngol Ital. 2014;34(5):299-309.

[34] D. R. Bielenberg and B. R. Zetter. The Contribution of Angiogenesis to the Process of Metastasis. Cancer J. (United States). 2015;21(4):267-273. doi: 10.1097/PPO.0000000000000138.

[35] J. Ma, H. Lu, S Wang, et al. The anthraquinone derivative emodin inhibits angiogenesis and metastasis through downregulating Runx2 activity in breast cancer. Int. J. Oncol. 2015;46(4):1619-1628. doi: 10.3892/ijo.2015.2888.

[36] M. Vassilakopoulou, A. Psyrri, and A. Argiris. Targeting angiogenesis in head and neck cancer. Oral Oncol. 2015;51(5):409-415. doi: 10.1016/j.oraloncology.2015.01.006.

[37] X. Zhang, M. Yamashita, H. Uetsuki, et al. Angiogenesis in renal cell carcinoma: Evaluation of microvessel density, vascular endothelial growth factor and matrix metalloproteinases. Int. J. Urol. 2002;9(9):509-514. doi: 10.1046/j.1442-2042.2002.00511.x.

[38] Y. Luo, D. Raible, J. Raper. Collapsin: A protein in brain thai induces the collapse and paralysis of neuronal growth cones. Cell. 1993;75(2):217-227. 
[39] L. Acevedo, S. Barillas, S. Weis et al. Semaphorin 3A suppresses VEGF- meidated angiogenesis yet acts as a vascular permeability factor. Blood. 2008;111(5):2674-2680.

[40] A. Sakurai, C. Doci, J. Gutkind. Semaphorin signaling in angiogenesis, lymphangiogenesis and cancer. Cell Res. 2012;22(1):23-32.

[41] F. MAione, S. Capano, D. Regano, et al. Semaphorin 3A overcomes cancer hypoxia and metastatic dissemination induced by antiangiogenic treatment in mice. J Clin Invest. 2012;122(5): 1832-1848.

[42] Ted Shid, PharmD, C. Lindley, eta al. Bevacizumab: An angiogenesis Inhibitor for the Treament of solid malignancies. Clin Ther. 2006;28(11):1779-1802.

\section{Tables}

Table 1. SEMA3A expression in normal tissues and OPC tissues

\begin{tabular}{|l|l|l|l|l|}
\hline \multirow{2}{*}{} & \multirow{2}{*}{ No. } & \multicolumn{3}{|l|}{ SEMA3A } \\
\cline { 3 - 5 } & & Low & High & $\boldsymbol{P}$ \\
\hline Tumor & 32 & 19 & 13 & 0.005 \\
\hline Normal & 17 & 3 & 14 & \\
\hline
\end{tabular}

(No., number of patients; L, low-expression cohort; H, high-expression cohort; P-value $<0.05$ was considered statistically significant)

Table 2. Relationship between SEMA3A expression in the tumor and clinical characteristics 


\begin{tabular}{|c|c|c|c|c|}
\hline \multirow[t]{2}{*}{ Characteristic } & & \multicolumn{3}{|c|}{ SEMA3A } \\
\hline & & Low & High & $P$ \\
\hline Sex & & & & 0.314 \\
\hline Male & 25 & 16 & 9 & \\
\hline Female & 7 & 3 & 4 & \\
\hline Age (years) & & & & 0.683 \\
\hline$\leq 50$ & 4 & 2 & 2 & \\
\hline$>50$ & 28 & 17 & 11 & \\
\hline Subsite & & & & 0.722 \\
\hline Lateral (tonsil and pillars) & 21 & 12 & 9 & \\
\hline $\begin{array}{l}\text { Frontal (base of tongue) } \\
\text { Upper (soft palate, uvula) } \\
\text { Posterior }\end{array}$ & 11 & 7 & 4 & \\
\hline TNM stage & & & & 0.654 \\
\hline I-II & 14 & 9 & 5 & \\
\hline III-IV & 16 & 9 & 7 & \\
\hline Tumor stage & & & & 0.757 \\
\hline T1-2 (early) & 19 & 11 & 8 & \\
\hline T3-4 (advanced) & 11 & 7 & 4 & \\
\hline Lymph node metastasis & & & & 0.543 \\
\hline N0 (negative) & 12 & 8 & 4 & \\
\hline N1-3 (positive) & 18 & 10 & 8 & \\
\hline HPV & & & & 0.821 \\
\hline Positive & 14 & 8 & 6 & \\
\hline Negative & 18 & 11 & 7 & \\
\hline Smoking & & & & 0.515 \\
\hline Never & 12 & 8 & 4 & \\
\hline Past and present & 20 & 11 & 9 & \\
\hline \multicolumn{5}{|l|}{ Alcohol } \\
\hline Never & 16 & 10 & 6 & 0.719 \\
\hline Past and present & 16 & 9 & 7 & \\
\hline
\end{tabular}

(L, low-expression cohort; $\mathrm{H}$, high-expression cohort; P-value < 0.05 was considered statistically significant) 


\section{Figures}

A

Fig. 1

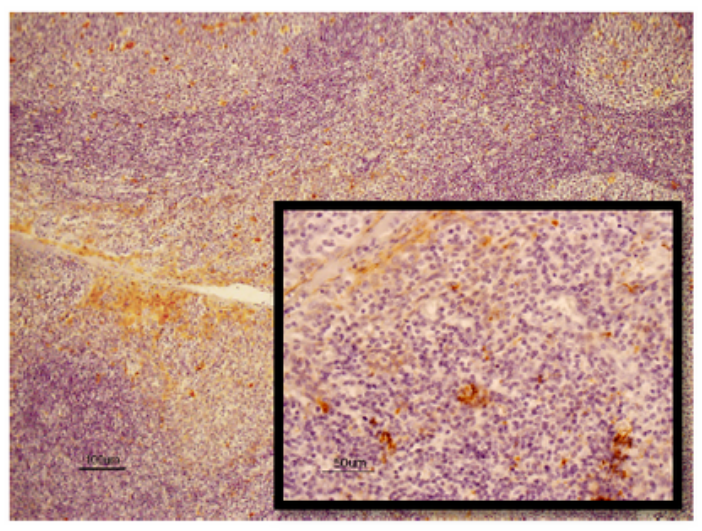

\section{B}

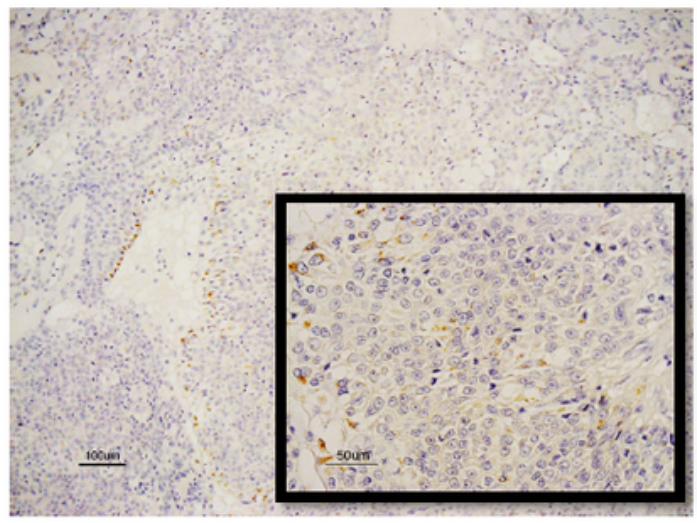

C

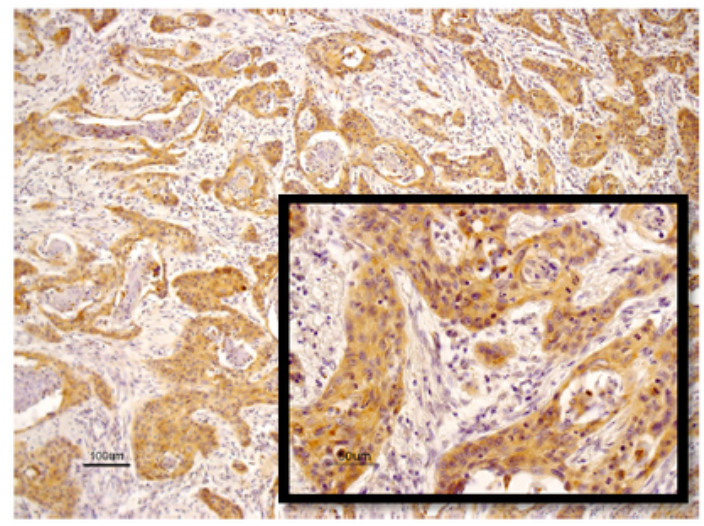

Figure 1 
a. SEMA3A in normal tonsil tissue (magnification $x 100$, lower right rectangle $x 400$ ) b. c. SEMA3A was detected primarily in the cytoplasm of tumor cells (magnification $x 100$, lower right rectangle $x 400$ ) b. Low level of SEMA3A expression c. High level of SEMA3A expression

Fig. 2
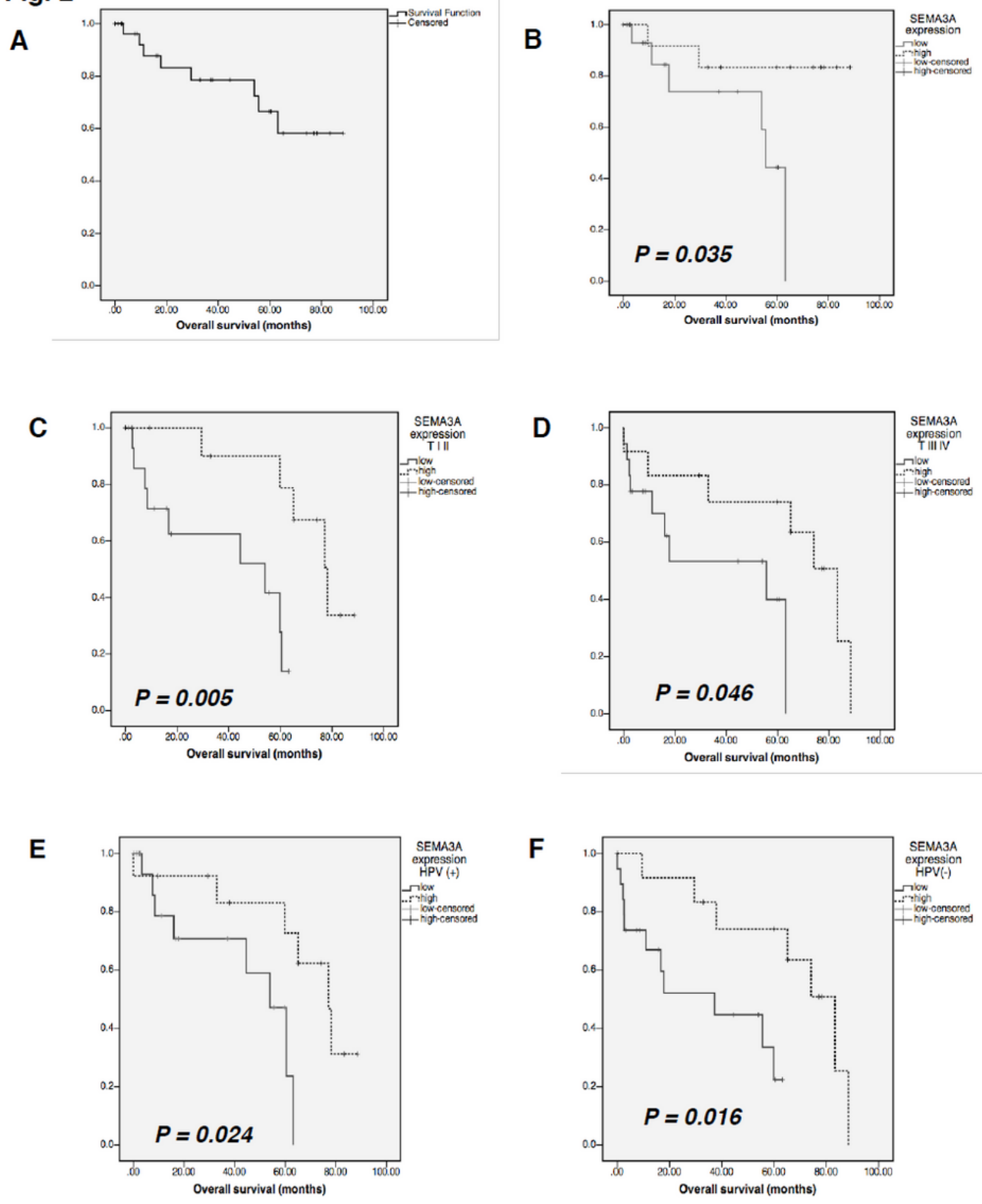

Figure 2

Comparison of different Kaplan-Meier curves for overall survival for patients grouped by immunohistochemistry levels of SEMA3A. a. Kaplan-Meier curves for overall survival (OS) of the 32 OPC 
patients. b. Kaplan-Meier curves for OS for OPC patients with low and high levels of SEMA3A expression $(P=0.035)$. c. Kaplan-Meier curves for OS for early-stage (stages I and II) OPC patients with low and high levels of SEMA3A expression ( $P=0.005$ ). d. Kaplan-Meier curves for OS for advanced stage (stages III and IV) OPC patients with low and high levels of SEMA3A expression $(P=0.046)$. e. Kaplan-Meier curves for OS in patients who were HPV $(+)$ with low and high levels of SEMA3A expression $(P=0.024)$. $f$. Kaplan-Meier curves for OS in patients who were HPV (-) with low and high levels of SEMA3A expression $(P=0.016)$.

\section{Fig. 3}

A

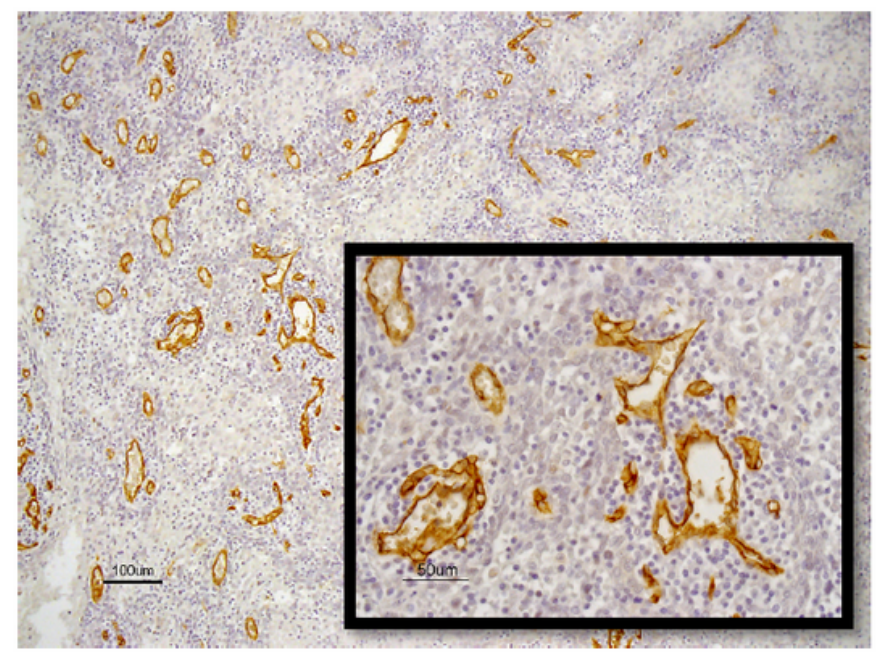

B

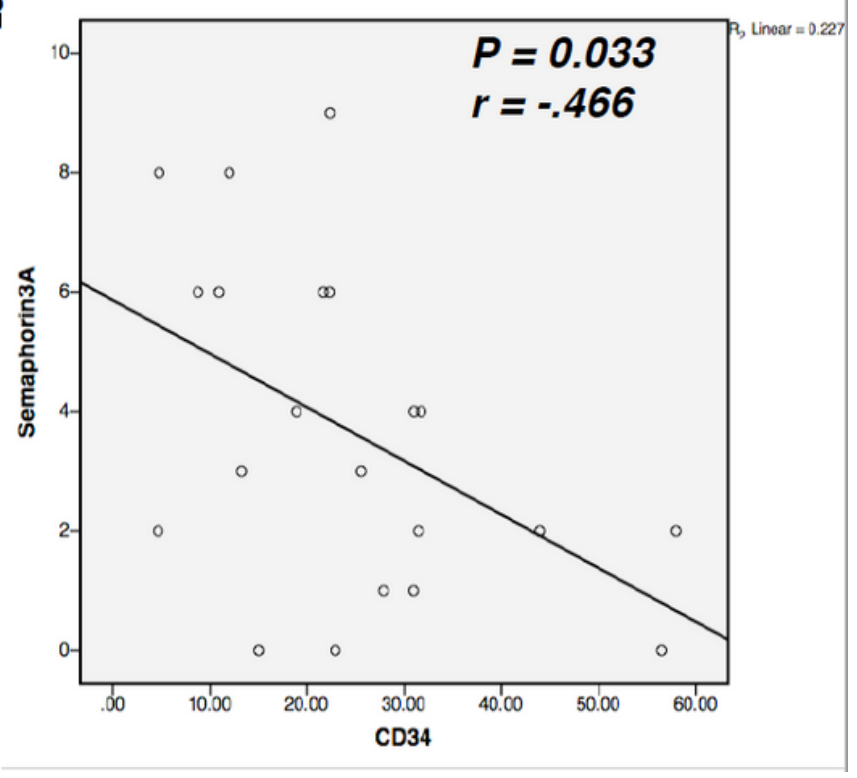

\section{Figure 3}

a. Immunohistochemical detection of CD34 protein expression in OPC tissues (magnification $x 100$, lower right rectangle $\mathrm{x} 400$ ) b. Relationship between SEMA3A expression and MVD. A Spearman correlation test was performed between SEMA3A expression and CD34 (microvessel density). There was a significant negative correlation between SEMA3A and CD34 $(P=0.033, r=-0.466)$. 